\title{
Quantum Electrodynamics of Casimir Momentum: Momentum of the Quantum Vacuum?
}

\author{
S. Kawka ${ }^{1}$ and B.A. van Tiggelen ${ }^{1}$ \\ ${ }^{1}$ Laboratoire de Physique et Modélisation des Milieux Condensés, \\ Université Joseph Fourier and CNRS, Maison des Magistères, BP 166, 38042 Grenoble, France
}

(Dated: November 3, 2009)

\begin{abstract}
The electromagnetic vacuum is known to have energy. It has been recently argued that the quantum vacuum can possess momentum, that adds up to the momentum of matter. This "Casimir momentum" is closely related to the Casimir effect, in which case energy is exchanged. In previous theory it was treated semi-classically. We present a non-relativistic quantum theory for the linear momentum of electromagnetic zero-point fluctuations, considering an harmonic oscillator subject to crossed, quasi-static magnetic and electric and coupled to the quantum vacuum. We derive a contribution of the quantum vacuum to the linear pseudo-momentum and give a new estimate for the achievable speed. Our analysis show that the effect exists and that it is finite.
\end{abstract}

Casimir energy refers to the electromagnetic (EM) energy that shows up when dielectric or metallic objects interact with the quantum vacuum. It is undoubtedly one of the most fascinating phenomena in physics, with a rich history in the $20 \mathrm{Th}$ century. Casimir forces become important on sub-micron scales and are thus believed to play an important role in nano-optics [1]. Casimir energy has been the subject of many speculations, such as its role in sonoluminescence [2] or in the cosmological constant problem [3].

The standard Casimir effect refers to the reduction of the EM zero-point energy when two ideal metallic plates approach [4]. Other well-known phenomena related to Casimir energy are Van Der Waals and Casimir-Polder forces between neutral atoms [5], the Lifshitz forces between dielectric media, and arguably the most famous among all, the Lamb shift of atomic levels. Shortly after its observation by Lamb in 1947 [6], Bethe explained the Lamb shift by the change in EM vacuum energy caused by the interaction of the atom with the quantum vacuum [7, 8]. The Lamb shifts in light atoms are now understood to be basically nonrelativistic QED phenomena, although full relativistic theory, including the contribution of several percents due to vacuum polarization, is necessary to come to the extraordinary agreement with experiment, unprecedented in physics. For the two-photon 1S-2S transition in atomic hydrogen, the shift is known up to several cycles [9].

Energy and momentum are naturally related by relativity. The search for "Casimir momentum" seems therefore obvious. In 2004 Feigel [10] proposed a quantum correction to the momentum of dielectric media exposed to static electric and magnetic fields. In this case, classical electrodynamics provides the following expression for the linear momentum of a neutral, polarizable object with mass $M$,

$$
\mathbf{Q}_{\text {class }}=M \mathbf{v}-\alpha(0) \mathbf{E}_{0} \times \mathbf{B}_{0}
$$

which is conserved in time, even if the external electric field $\mathbf{E}_{0}$ is varied slowly in time. Here $\alpha(0)$ is the static polarizability, with the dimension of a volume. The semiclassical theory of Ref.[10] predicts a strongly diverging contribution of the quantum vacuum to Eq. (1), quite similar to the one encountered for Casimir energy. Fortunately, spatial gradients of Casimir energy - observable as forces - are often found not to diverge. Momentum however is an observable parameter and the divergence does pose a problem. It has been suggested that UV divergences are not physical and should disappear into the values attributed to physical observables, such as inertial mass, electric charge or cosmological constant [11]. If this is true it is not evident that the prediction of "Casimir momentum" found in Ref.[10] will survive or be measurable. An obvious next question is what physical observable will then absorb the UV divergence of Casimir momentum. In this work we provide first answers to these questions. We use the method of mass renormalization first employed by Bethe and Kramers that results in a finite Casimir momentum of simple quantum objects.

It is now realized that Casimir momentum emerges quite generally in so-called bi-anisotropic media, in which also magnetic fields can induce an electric polarization [12, 13]. Except in media exposed to external EM fields, bi-anisotropy also occurs in moving dielectric media. This follows from the relativistic transformations of EM fields, and shall be discussed elsewhere [14]. In general, like spin, bi-anisotropic behavior and Casimir momentum can be viewed as "remnants" of special relativity [15] in non-relativistic theory that often suffices to describe phenomena quantitatively.

We consider here the following system: a 3D harmonic oscillator - composed of two particles with opposite charge $q_{1}=+e$ and $q_{2}=-e$ and masses $m_{i}$ - exposed to crossed, homogeneous static EM fields $\mathbf{E}_{0}, \mathbf{B}_{0}$ which constitute our bi-anisotropic object, coupled to the EM vacuum. In the Schrödinger picture the Hamiltonian is 
given by

$$
\begin{aligned}
H & =\sum_{i=1}^{2}\left[\frac{1}{2 m_{i}}\left(\mathbf{p}_{i}-q_{i} \mathbf{A}_{t}\left(\mathbf{r}_{i}\right)\right)^{2}-q_{i} \mathbf{E}_{0} \cdot \mathbf{r}_{i}\right]+V(\mathbf{r}) \\
& +\sum_{\mathbf{k} \epsilon} \hbar \omega_{k}\left[a_{\mathbf{k} \epsilon}^{\dagger} a_{\mathbf{k} \epsilon}+\frac{1}{2}\right]
\end{aligned}
$$

where $\mathbf{A}_{t}=\mathbf{A}_{0}+\mathbf{A}$ is the total vector potential containing a contribution from the external, static, classical magnetic field, described by the classical vector potential $\mathbf{A}_{0}(\mathbf{r})=\frac{1}{2} \mathbf{B}_{0} \times \mathbf{r}$, and the quantum operator $\mathbf{A}$ of the EM field. The EM bath will be treated in the Coulomb gauge. We will use $\mathbf{R}=\left(m_{1} \mathbf{r}_{1}+m_{2} \mathbf{r}_{2}\right) / M$ and $\mathbf{r}=\mathbf{r}_{1}-\mathbf{r}_{2}$ for the center of mass position and the inter-particle distance, with conjugate momenta $\mathbf{P}$ and $\mathbf{p}$, respectively; $M=m_{1}+m_{2}$ and $\mu=\left(1 / m_{1}+1 / m_{2}\right)^{-1}$ are the total and reduced mass. For a harmonic oscillator we can then write $V(\mathbf{r})=\frac{1}{2} \mu \omega_{0}^{2} \mathbf{r}^{2}$.

As we are looking for momentum, we notice the existence of a conserved pseudo-momentum $\mathbf{K}$ that commutes with $H$ [17], even when the electric field is varied in time. The electric field is a parameter that can be varied experimentally. This momentum has contributions from both atom and radiation,

$$
\mathbf{K}=\mathbf{P}+\frac{e}{2} \mathbf{B}_{0} \times \mathbf{r}+\sum_{\mathbf{k} \epsilon} \hbar \mathbf{k}\left[a_{\mathbf{k} \epsilon}^{\dagger} a_{\mathbf{k} \epsilon}+\frac{1}{2}\right]
$$

It is a pseudo-momentum as it is a constant of the motion only if the external magnetic field $\mathbf{B}_{0}$ is time independent and homogeneous, as we will assume here. Note that we are interested in the change of the total kinetic momentum $\mathbf{P}_{\text {kin }}$ of the oscillator in the presence of vacuum, which is not equal to $\mathbf{K}$, neither to $\mathbf{P}$. Yet, because $\mathbf{K}$ is conserved, even for a slowly time-dependent electric field $\mathbf{E}_{0}$, it is the appropriate momentum to look at. In the presence of magnetic fields the kinetic and conjugate momenta operators are related by $\mathbf{P}_{\text {kin }}=\mathbf{P}-e \Delta \mathbf{A}_{t}$ with $e \Delta \mathbf{A}_{t}=e \mathbf{A}_{t}\left(\mathbf{r}_{1}\right)-e \mathbf{A}_{t}\left(\mathbf{r}_{2}\right)$, so that we can obtain

$$
\mathbf{K}=\mathbf{P}_{\text {kin }}+e \mathbf{B}_{0} \times \mathbf{r}+e \Delta \mathbf{A}+\sum_{\mathbf{k} \epsilon} \hbar \mathbf{k}\left[a_{\mathbf{k} \epsilon}^{\dagger} a_{\mathbf{k} \epsilon}+\frac{1}{2}\right]
$$

The operator $e \Delta \mathbf{A}=e \mathbf{A}\left(\mathbf{r}_{1}\right)-e \mathbf{A}\left(\mathbf{r}_{2}\right)$ guarantees the gauge-invariant contribution of the "longitudinal" vacuum field to the pseudo-momentum, in terms of the vector potential $\mathbf{A}(\mathbf{r})$ quantized as usual inside a quantization volume $V, \mathbf{A}(\mathbf{r})=\sum_{\mathbf{k} \epsilon} \mathcal{A}_{\mathbf{k}} \boldsymbol{\epsilon}\left[a_{\mathbf{k} \epsilon} e^{i \mathbf{k r}}+a_{\mathbf{k} \epsilon}^{\dagger} e^{-i \mathbf{k r}}\right]$. We will be obliged to go beyond the electric dipole approximation -in which $e \Delta \mathbf{A}$ would be neglected- to treat the high wave numbers of zero-point fluctuations accurately. The last term in Eq. (44) stems from the "transverse" electromagnetic field in the vicinity of the atom 17].
We wish to express the expectation value $\overline{\mathbf{K}}=$ $\left\langle\Psi_{0}|\mathbf{K}| \Psi_{0}\right\rangle$, of the pseudo-momentum in the total ground state $\left|\Psi_{0}\right\rangle$ in terms of the two relevant vectors: the kinetic momentum $M \mathbf{v}$ of the oscillator and the magneto-electric vector $\mathbf{E}_{0} \times \mathbf{B}_{0}$. We will use perturbation theory in the coupling between the magnetoelectric oscillator and the EM field, where the small parameter of the expansion is the fine structure constant $\alpha$. To lowest order, only the emission and subsequent re-absorption of one virtual photon will contribute at this one-loop level of the theory, which will thus be second order perturbation theory. In order to facilitate perturbation theory, we split the Hamiltonian (2) up as follow,

$$
\begin{gathered}
H=H_{0}+H_{F}+W \\
H_{0}=\sum_{i=1}^{2}\left[\frac{1}{2 m_{i}}\left(\mathbf{p}_{i}-q_{i} \mathbf{A}_{0}\left(\mathbf{r}_{i}\right)\right)^{2}-q_{i} \mathbf{E}_{0} \cdot \mathbf{r}_{i}\right]+\frac{1}{2} \mu \omega_{0}^{2} \mathbf{r}^{2} \\
H_{F}=\sum_{\mathbf{k} \epsilon} \hbar \omega_{k}\left[a_{\mathbf{k} \epsilon}^{\dagger} a_{\mathbf{k} \epsilon}+\frac{1}{2}\right] \\
W=\sum_{i=1}^{2}-\frac{q_{i}}{m_{i}}\left(\mathbf{p}_{i}-q_{i} \mathbf{A}_{0}\left(\mathbf{r}_{i}\right)\right) \mathbf{A}\left(\mathbf{r}_{i}\right)+\frac{q_{i}^{2}}{2 m_{i}} \mathbf{A}\left(\mathbf{r}_{i}\right)^{2}
\end{gathered}
$$

The operator $W$ represents the perturbation of the quantum vacuum on the atom. The term $\mathbf{A}^{2}$ can be disregarded because it does not couple field and matter, and at this order its contribution to $\overline{\mathbf{K}}$ will vanish due to basic selection rules. The photon field in free space described by $H_{F}$ is well known. Finally, the quantum-mechanics of a 3D harmonic oscillator exposed to crossed, homogeneous static EM fields, described by $H_{0}$, was discussed in detail and non-perturbationally by Dippel etal [16]. $H_{0}$ and $H_{F}$ act in different Hilbert-spaces and the basis will be the direct product of their eigenvectors. As we are interested in the center-of-mass motion of the 3D harmonic oscillator, one key point is the distinction between the center-of-mass and the internal coordinates. Without the quantum vacuum, the pseudo-momentum $\mathbf{K}$ reduces to,

$$
\mathbf{Q}=\mathbf{P}_{\text {kin }}+e \mathbf{B}_{0} \times \mathbf{r}
$$

which commutes with the atomic Hamiltonian. $\mathbf{Q}$ is the quantum-mechanical operator corresponding to the classical pseudo-momentum $\mathbf{Q}_{\text {class }}$ in Eq. (1). For the purpose of this work it is convenient to choose eigenfunctions that simultaneously diagonalize the atomic Hamiltonian and the pseudo-momentum $\mathbf{Q}$, labeled by the eigenvalue $\mathbf{Q}_{0}$, as $\mathbf{Q}$ appears directly in $\mathbf{K}$ as seen by inserting Eq. (4) into Eq. (6). It will be sufficient to ignore all contributions other than on those linear in either $\mathbf{E}_{0}$ and $\mathbf{B}_{0}$, as indicated by the sign $\asymp$. In this approximation, the magneto-electric oscillator is unitary equivalent to an isotropic harmonic oscillator as expressed by, 


$$
\left|\mathbf{n}, \mathbf{Q}_{0}\right\rangle \asymp \exp \left(\frac{i}{\hbar} \mathbf{Q}_{0} \cdot \mathbf{R}\right) \exp \left[-\frac{i}{2 \hbar}\left(\mathbf{B}_{0} \times \mathbf{r}\right) \cdot \mathbf{R}\right] \exp \left(-\frac{i}{\hbar} \mathbf{p}_{0} \cdot \mathbf{r}\right) \exp \left(-\frac{i}{\hbar} \mathbf{p} \cdot \mathbf{r}_{0}\right)\left|\phi_{\mathbf{n}}\right\rangle
$$

$\mathbf{n}=\left(n_{x}, n_{y}, n_{z}\right), n_{i}=0,1, \cdots$ denotes the quantum levels of the oscillator. The first two exponentials on the right denote translational momentum of the center of mass, and governed by the conjugate momentum $\mathbf{P}$. The last pair of exponentials eliminate the static electric field from the picture, with the eigenfunctions of the oscillator shifted out of the center of mass over a distance $\mathbf{r}_{0}=e^{-1} \alpha(0)\left(\mathbf{E}_{0}+\mathbf{Q}_{0} \times \mathbf{B}_{0} / M\right)$, and the reduced momentum shifted by $\mathbf{p}_{0} \asymp(2 M)^{-1}\left(m_{2}-m_{1}\right) \alpha(0)\left(\mathbf{E}_{0} \times \mathbf{B}_{0}\right)$ with $\alpha(0)=e^{2} / \mu \omega_{0}^{2}$ the static polarizability of the oscillator. This last feature is reminiscent of bi-anisotropic activity, and will generate a dominant contribution to $\overline{\mathbf{K}}$. Note that it vanishes for $m_{1}=m_{2}$. Due to the static magnetic field, the oscillator states $\left|\phi_{\mathbf{n}}\right\rangle$ are in principle still anisotropic and even in $B_{0}$. The anisotropy is estimated by the small parameter $e B_{0} a /(\hbar / a) \approx 10^{-5}$, with $a$ the atomic size. This anisotropy constitutes corrections nonlinear in the applied fields to the final result for the total momentum. We can therefore neglect it.

Upon taking the quantum-expectation value of Eq. (6) for the atomic ground state, which still ignores the quantum vacuum, reveals that the eigenvalue $\mathbf{Q}_{0}$ is just equal to the classical expression (11). The total energy of the oscillator in the ground state $E_{0} \asymp \frac{3}{2} \hbar \omega_{0}+Q_{0}^{2} / 2 M$ is minimal when $\mathbf{Q}_{0}=0$, i.e. for a finite kinetic momentum.

In the absence of the interaction with the quantum vacuum, the eigenstates are just the direct products $\left|\mathbf{n}, \mathbf{Q}_{0}, \mathbf{n}_{\mathbf{k}}\right\rangle=\left|\mathbf{n}, \mathbf{Q}_{0}\right\rangle \otimes\left|\mathbf{n}_{\mathbf{k}}\right\rangle$, with unperturbed energies $E_{\mathbf{n} \mathbf{Q}_{0} \mathbf{n}_{\mathbf{k}}}=E_{\mathbf{n} \mathbf{Q}_{0}}+\sum_{\mathbf{k}} \hbar \omega_{\mathbf{k}}\left(n_{\mathbf{k}}+\frac{1}{2}\right)$. Here $\mathbf{n}_{\mathbf{k}}$ is the occupation of the EM Fock states with photon momentum $\hbar \mathbf{k}$. The ground state $\left|\Psi_{0}\right\rangle$ follows from second-order perturbation in the coupling $W$ to the quantum vacuum $\left(\Sigma^{\prime}\right.$ avoids zeros in the denominator),

$$
\begin{aligned}
& \left|\Psi_{0}\right\rangle=\left|\mathbf{0}, \mathbf{Q}_{0}, \mathbf{0}\right\rangle+\sum_{\mathrm{lQn}}^{\prime} \frac{W_{\mathrm{lQn}, 0 \mathbf{Q}_{0} \mathbf{0}}}{E_{\mathbf{0} \mathbf{Q}_{0} \mathbf{0}}-E_{\mathrm{lQn}}}|\mathbf{l}, \mathbf{Q}, \mathbf{n}\rangle \\
& +\sum_{\mathbf{l} \mathbf{Q n}}^{\prime} \sum_{\mathbf{s} \mathbf{Q}^{\prime} \mathbf{m}}^{\prime} \frac{W_{\mathbf{l Q n}, \mathbf{s} \mathbf{Q}^{\prime} \mathbf{m}} W_{\mathbf{s} \mathbf{Q}^{\prime} \mathbf{m}, \mathbf{0} \mathbf{Q}_{0} \mathbf{0}}}{\left(E_{\mathbf{0} \mathbf{Q}_{0} \mathbf{0}}-E_{\mathbf{l Q n}}\right)\left(E_{\mathbf{0} \mathbf{Q}_{0} \mathbf{0}}-E_{\mathbf{s} \mathbf{Q}^{\prime} \mathbf{m}}\right)}|\mathbf{l}, \mathbf{Q}, \mathbf{n}\rangle \\
& +\sum_{\mathbf{l Q n}}^{\prime} \frac{\left|W_{\mathbf{l Q n}, 0 \mathbf{Q}_{0} \mathbf{0}}\right|^{2}}{\left(E_{\mathbf{0} \mathbf{Q}_{0} \mathbf{0}}-E_{\mathbf{l Q n}}\right)^{2}}|\mathbf{l}, \mathbf{Q}, \mathbf{n}\rangle
\end{aligned}
$$

Only the emission and subsequent re-absorption of one virtual photon is considered, which generates a tempo- rary recoil momentum $\mathbf{Q}_{0}-\hbar \mathbf{k}$ of the oscillator,

$$
\begin{aligned}
& \overline{\mathbf{K}}=\mathbf{Q}_{0} \\
& +e^{2} 2 \operatorname{Re} \sum_{\mathbf{l} \mathbf{\epsilon} \epsilon} \mathcal{A}_{\mathbf{k}}^{2} \epsilon \frac{\left\langle\phi_{\mathbf{0}}\left|\left(e^{i \mathbf{k}\left(\mathbf{r}+\mathbf{r}_{0}\right) \frac{m_{2}}{M}}-e^{-i \mathbf{k}\left(\mathbf{r}+\mathbf{r}_{0}\right) \frac{m_{1}}{M}}\right)\right| \phi_{\mathbf{l}}\right\rangle \Omega_{\mathbf{1}, \mathbf{0}}^{*}}{E_{\mathbf{0} \mathbf{Q}_{0} \mathbf{0}}-E_{\mathbf{l}\left(\mathbf{Q}_{0}-\hbar \mathbf{k}\right) 1_{k}}} \\
& +\mathbf{B}_{0} \times 2 \operatorname{Re} \sum_{\mathbf{l s k} \boldsymbol{\epsilon}} \frac{e^{3} \mathcal{A}_{\mathbf{k}}^{2}\left\langle\phi_{\mathbf{0}}\left|\mathbf{r}+\mathbf{r}_{0}\right| \phi_{\mathbf{l}}\right\rangle \Omega_{\mathbf{1}, \mathbf{s}} \Omega_{\mathbf{s}, \mathbf{0}}^{*}}{\left(E_{\mathbf{0} \mathbf{Q}_{0} \mathbf{0}}-E_{\mathbf{l} \mathbf{Q}_{0} \mathbf{0}}\right)\left(E_{\mathbf{0} \mathbf{Q}_{0} \mathbf{0}}-E_{\mathbf{s}\left(\mathbf{Q}_{0}-\hbar \mathbf{k}\right) 1_{k}}\right)} \\
& +\mathbf{B}_{0} \times \sum_{\mathbf{l s k} \epsilon} \frac{e^{3} \mathcal{A}_{\mathbf{k}}^{2}\left\langle\phi_{\mathbf{s}}\left|\mathbf{r}+\mathbf{r}_{0}\right| \phi_{\mathbf{l}}\right\rangle \Omega_{\mathbf{l}, \mathbf{0}}^{*} \Omega_{\mathbf{0}, \mathbf{s}}}{\left(E_{\mathbf{0} \mathbf{Q}_{0} \mathbf{0}}-E_{\mathbf{l}\left(\mathbf{Q}_{0}-\hbar \mathbf{k}\right) 1_{k}}\right)\left(E_{\mathbf{0} \mathbf{Q}_{0} \mathbf{0}}-E_{\mathbf{s}\left(\mathbf{Q}_{0}-\hbar \mathbf{k}\right) 1_{k}}\right)}
\end{aligned}
$$

The second term on the right-hand-side is due to $e \Delta \mathbf{A}$ in Eq. (4), the two last stem from $e \mathbf{B}_{0} \times \mathbf{r}$. We introduced the matrix element $\Omega_{1, \mathrm{~s}}=\left\langle\phi_{\mathbf{1}}|\Omega| \phi_{\mathrm{s}}\right\rangle$ of the operator

$$
\begin{aligned}
\Omega & =e \boldsymbol{\epsilon} \cdot\left[\mathbf{B}_{0} \times\left(\mathbf{r}+\mathbf{r}_{0}\right)\right]\left(\frac{e^{i \mathbf{k}\left(\mathbf{r}+\mathbf{r}_{0}\right) \frac{m_{2}}{M}}}{m_{1}}-\frac{e^{-i \mathbf{k}\left(\mathbf{r}+\mathbf{r}_{0}\right) \frac{m_{1}}{M}}}{m_{2}}\right) \\
& -\frac{\mathbf{Q}_{0}}{M} \cdot \boldsymbol{\epsilon}\left(e^{i \mathbf{k}\left(\mathbf{r}+\mathbf{r}_{0}\right) \frac{m_{2}}{M}}-e^{-i \mathbf{k}\left(\mathbf{r}+\mathbf{r}_{0}\right) \frac{m_{1}}{M}}\right) \\
& -\left(\mathbf{p}-\mathbf{p}_{0}\right) \cdot \boldsymbol{\epsilon}\left(\frac{e^{i \mathbf{k}\left(\mathbf{r}+\mathbf{r}_{0}\right) \frac{m_{2}}{M}}}{m_{1}}+\frac{e^{-i \mathbf{k}\left(\mathbf{r}+\mathbf{r}_{0}\right) \frac{m_{1}}{M}}}{m_{2}}\right)
\end{aligned}
$$

and $\mathcal{A}_{\mathbf{k}}^{2}=\hbar / 2 \varepsilon_{0} V k c$ familiar from quantum optics. $\Omega$ stems directly from the development of $W$ applied to a one photon transition.

Three kinds of contributions to $\overline{\mathbf{K}}$ can be identified. The first class is proportional to $\mathbf{Q}_{0}$ (see the middle term in $\Omega_{1, \mathbf{s}}$ ) that survives even in the absence of external fields. This term can be seen to affect the inertial mass of the atom, by typically $\frac{3}{2} \frac{\hbar \omega_{0}}{c^{2}}$ in accordance with the equivalence principle of energy and inertia [14]. Its UV divergence can be absorbed into the total mass $M$ in the same way as will be discussed below for the ME divergences.

The second class, represented by the two last contributions in Eq. (8), are actually QED contributions to the induced electrical dipole moment $\left\langle\Psi_{0}|e r| \Psi_{0}\right\rangle$ of the oscillator, that find their way to the total momentum via the classical expression (11). It is straightforward to calculate these corrections - they actually do not diverge and they are relatively small - but we note that if an experimental value for $\alpha(0)$ is used to evaluate the "classical" contribution, these terms are automatically included. In this sense they do not constitute a genuine "Casimir momentum".

The term $e \Delta \mathbf{A}$ in Eq. (4) is a genuine contribution of the vacuum radiation to the pseudo-momentum. It will be seen to generate a momentum linear in $\mathbf{E}_{0} \times \mathbf{B}_{0}$ by 
means of the second term in Eq. (8) and the third term in $\Omega$. The following calculation will focus on this contribution. It suffers from a UV divergence, that can be eliminated by exactly the same mass regularization as applied by Bethe in his calculation of the Lamb shift [7]. In particular, this procedure establishes that the reduced mass featuring in the static polarizability $\alpha(0)=e^{2} / \mu \omega_{0}^{2}$ will be replaced by the "observed" reduced mass. It is straightforward to show that the so-called transverse electromagnetic momentum, represented by the last term of Eq. (4), does not contribute a net momentum. This follows from selection rules and spatial symmetry.

We apply the closure relation $\sum_{\mathbf{l}}\left|\phi_{\mathbf{l}}\right\rangle\left\langle\phi_{\mathbf{l}}\right|\left(E(k)+E_{\mathbf{l}}\right)^{-1}=\left(E(k)+H_{h o}\right)^{-1}$ in the second contribution to $\overline{\mathbf{K}}$. This generates two terms involving exponentials with opposite phases that cancel. The contribution from the first term in $\Omega$ to $\overline{\mathbf{K}}$ is

$$
\alpha(0) \mathbf{E}_{0} \times \mathbf{B}_{0} \frac{4 \alpha}{3 \pi}\left[\frac{\hbar^{2}}{m_{i}} \int \frac{k d k}{\frac{\hbar^{2} k^{2}}{2 m_{i}}+\hbar c k}+\mathcal{O}\left(\frac{\hbar \omega_{0}}{M c^{2}}\right)\right]
$$

with $i=1,2$. Here we neglect in the denominator the Doppler terms $\mathbf{Q}_{0} \cdot \hbar \mathbf{k}$ and $\mathbf{p} \cdot \hbar \mathbf{k}$, generated by the identity $e^{i \mathbf{k r}} H_{h o}(\mathbf{p}) e^{-i \mathbf{k r}}=H_{h o}(\mathbf{p}-\hbar \mathbf{k})$. They provide a finite correction of order $\frac{\hbar \omega_{0}}{M c^{2}} \sim 10^{-8}$. The leading contribution diverges logarithmically. In the Bethe theory for the Lamb shift [7] exactly the same kind of divergency was encountered. The two diverging mass-like terms $\delta m_{i}=\frac{4 \alpha}{3 \pi} \hbar^{2} \int k d k\left(\frac{\hbar^{2} k^{2}}{2 m_{i}}+\hbar c k\right)^{-1}$ - here with recoil effects included - stem from the QED coupling of the free particles 1 and 2 with the quantum vacuum and are therefore naturally interpreted to be part of their intrinsic, observable masses [8]. Two other diverging contributions are generated by the term $\alpha(0) \mathbf{E}_{0} \times \mathbf{B}_{0}$ contained in $\mathbf{Q}_{0}$, see (1), in the expression for $\Omega$ and add up to $-\delta m_{1} / M-\delta m_{2} / M=-\delta M / M$. Adding up all diverging terms we obtain

$\mu^{-1}\left(\delta m_{1} / m_{1}+\delta m_{2} / m_{2}-\delta M / M\right)=\mu^{-2} \delta \mu=-\delta(1 / \mu)$

Since the static polarizability is proportional to $1 / \mu$, these UV-divergent corrections all disappear into the factor $\alpha(0)$ of Eq. (1), which thus becomes defined in terms of the observed reduced mass $\mu^{*}$ :

$$
\mathbf{Q}_{0}-\delta\left(\frac{1}{\mu}\right) \frac{e^{2}}{\omega_{0}^{2}} \mathbf{E}_{0} \times \mathbf{B}_{0}=M \mathbf{v}-\frac{e^{2}}{\mu^{*} \omega_{0}^{2}} \mathbf{E}_{0} \times \mathbf{B}_{0}
$$

All other terms generated by Eq. (8) are finite. In particular, the term involving $\mathbf{p}_{0}$ in $\Omega$ generates the following contribution to $\mathbf{K}$ :

$$
\begin{aligned}
\mathbf{K}_{1} & =\alpha(0) \mathbf{E}_{0} \times \mathbf{B}_{0} \frac{m_{2}-m_{1}}{2 M} \frac{4 \alpha}{3 \pi} \\
& \times \lim _{\delta \downarrow 0} \int_{\delta}^{\infty} d k\left(\frac{k}{k^{2} / 2+c k m_{2} / \hbar}-\frac{k}{k^{2} / 2+\hbar c k m_{1} / \hbar}\right) \\
& =-\alpha(0) \mathbf{E}_{0} \times \mathbf{B}_{0} \frac{4 \alpha}{3 \pi} \frac{m_{1}-m_{2}}{M} \log \frac{m_{1}}{m_{2}} .
\end{aligned}
$$

The UV divergency cancels out, and the integral is finite. Of course part of the k-integral enters the relativistic regime $\hbar k>m_{i} c$ in which the present theory is not valid. However, by subtracting and adding $2 / k$ to both integrands in Eq. (10) reveals two terms whose range of integration is typically $m_{i} c / \hbar$. This wavenumber was used by Bethe to cut off his nonrelativistic theory for the Lamb shift [7]. We will thus adopt the final result in Eq. (10) as a "reasonable" nonrelativistic estimate for the Casimir momentum. It is nevertheless clear that a relativistic theory is required to get the complete picture for Casimir momentum.

All other cross-terms in Eq. (8) contain oscillating exponential factors and converge rapidly for $k>1 / a$, i.e. stay in the nonrelativistic regime. They generate a Casimir momentum that is typically a factor $\sqrt{\hbar \omega_{0} / \mu c^{2}} \sim \alpha$ smaller. We obtain,

$$
\begin{aligned}
\mathbf{K}_{2} & =\alpha(0) \mathbf{E}_{0} \times \mathbf{B}_{0} \alpha \sqrt{\frac{\hbar \omega_{0}}{\mu c^{2}}} \\
& \times\left(-\frac{14}{15 \sqrt{\pi}}+\frac{2}{3 \sqrt{\pi}}\left(\frac{\Delta m}{M}\right)^{2}+\frac{8}{3 \sqrt{\pi}} \frac{\mu}{M}\right)
\end{aligned}
$$

When both masses are equal, $\mathbf{K}_{2}$ becomes the sole contribution to Casimir momentum, with a relative correction of order $\alpha^{2}$. For $m_{1} \gg m_{2}, \mathbf{K}_{1}$ dominates. Since it is independent on details of the force between the two particles, it is tempting to apply Eq. (10) to the hydrogen atom. With $\hbar \omega_{0}=10 \mathrm{eV}, m_{1}=m_{p}$ and $m_{2}=m_{e}$, $E_{0}=10^{5} \mathrm{~V} / \mathrm{m}$ and $B_{0}=17 \mathrm{~T}$, we find for the velocity associated with the classical contribution (1) $v_{c l} \approx 5 \mu \mathrm{m} / \mathrm{s}$, and a QED correction of $2 \%$ in the same direction; $\mathbf{K}_{2}$ yields a negligible correction of $0.01 \%$.

In conclusion, we have presented a non-relativistic quantum electrodynamic theory for the total of an harmonic oscillator, subject to external classical fields, and coupled to the electromagnetic quantum vacuum. The most important conclusions of this work are that Casimir momentum exists and that its UV divergences are renormalizable. The theory shows it to be basically a nonrelativistic quantity, but that relativistic corrections are likely to be significant, much like in the Lamb shift problem. To our knowledge nor the classical contribution to magneto-electric momentum, neither the QED correction have ever been observed.

We are indebted to Geert Rikken for many helpful discussions. This work was supported by the contract PHOTONIMPULS ANR-09-BLAN-0088-01.

[1] See: A. Lambrecht, Physics World 15, 29 (2002).

[2] J. Schwinger, Proc. Natl. Acad. Sci. USA 91, 6473 (1994); C. Eberlein, Phys. Rev. A 53, 2772 (1996); A. 
Lambrecht, M.T. Jaekel, and S. Reynaud, Phys. Rev. Lett. 78, 2267 (1997).

[3] N.D. Birell and P.C.W. Davies, Quantum Fields in Curved Space (Cambridge, 1984).

[4] H.B.G. Casimir, Proc. Kon. Ned. Akad. Wetensch. 51, 793 (1948).

[5] H.B.G. Casimir, D. Polder, Phys. Rev. 73, 360 (1948).

[6] W.E. Lamb Jr. and R.C. Retherford, Phys. Rev. 72, 241 (1947).

[7] H. A. Bethe, Phys. Rev. 72, 339 (1947); see also P.W. Milonni, Phys. Rep. 25, 1 (1976).

[8] P.W. Milonni, The Quantum Vacuum (Academic Press, San Diego, 1994).

[9] C. Zimmermann, R. Kallenback and T.W. Hänsch, Phys.
Rev. Lett. 65, 571 (1990).

[10] A. Feigel, Phys. Rev. Lett. 92, 020404 (2004).

[11] K.A. Milton, J. Phys. A. 37, R209 (2004).

[12] O.J. Birkeland and I. Brevik, Phys. Rev. E 76, 066605 (2007).

[13] B.A. van Tiggelen, Eur. Phys. J. D 47, 261 (2008).

[14] S. Kawka and B.A. van Tiggelen, submitted.

[15] B.A. van Tiggelen, G.L.J.A. Rikken, Phys. Rev. Lett. 93, 268903 (2004).

[16] O. Dippel, P. Schmelcher, L.S. Cederbaum, Phys. Rev. A. 49, 4415 (1994).

[17] C. Cohen-Tannoudji, J. Dupont-Roc, G. Grynberg, Photons et atomes (Editions du CNRS, Paris, 1987). 